\title{
Stop (mis)classifying fractures as high- or low-trauma or as fragility fractures
}

\author{
S. R. Cummings ${ }^{1} \cdot$ R. Eastell ${ }^{2}$
}

Received: 9 January 2020 / Accepted: 28 January 2020 / Published online: 16 March 2020

(C) International Osteoporosis Foundation and National Osteoporosis Foundation 2020

We strongly recommend that caregivers for fractures stop classifying fractures as high- or low-trauma, fragility fractures and provide the same care for fractures now mislabeled as "high trauma" as all other fractures. The study by Leslie and colleagues in the current issue of Osteoporosis International adds to the evidence that attempts to classify the degree of trauma deprive a large number of patients with fractures of appropriate evaluation and preventive treatment [1].

The study adds evidence to the case that it is time to abandon the mistaken beliefs that fractures rated as high trauma are not associated with decreased BMD, indicate no higher risk of subsequent fracture, or are less likely to be prevented by treatments for osteoporosis. Rather, the evidence shows that "hightrauma" fractures are associated with decreased BMD and increased risk of future fracture and reduction in risk by treatment.

Fractures occur when, at skeletal site, the force of a trauma exceeds the strength of the bone. It seemed reasonable to posit that there is a degree force that exceeds the strength of any known bone. However, when an individual has suffered a fracture, it is not possible to retrospectively quantify the force of trauma or the strength of bone where the fracture occurred. Consider a fracture occurring during a car crash. The crash generates a wide array of forces that hit a body in innumerable possible locations, at different angles, where bones are variably protected by soft tissue and have different strengths at the point of impact. It is not possible to quantify array of forces generated by trauma "greater - or less - than generated by

S. R. Cummings

scummings@sfcc-cpmc.net

1 California Pacific Medical Center Research Institute, Mission Hall, 550-16th Street, 2nd Floor, San Francisco, CA, USA

2 Department of Oncology and Metabolism, University of Sheffield, Sheffield, UK falling from more than standing height" as often used to classify fractures as high-trauma or fragility fractures.

Even if it were possible to retrospectively estimate the degree of force that was applied to the bone that fractured, it would be true that given the same amount of trauma - such as a crash - the probability of fracture in any skeletal location will increase as bone strength decreases. If so, then, individuals who have fractures clinically classified as "traumatic" or "high-trauma" should have lower bone strength than those who have not had such fractures. Thus, those with a fracture resulting from a high-trauma event should have a greater risk of future fractures - both "low-" and "high-"trauma fractures. They should also have a lower average bone density. These suppositions have been supported by previous studies [2, 3].

The study by Leslie and colleagues in this issue of Osteoporosis International found that people with prior hightrauma fracture had BMD reduced to the same extent as those with prior low-trauma fracture and similar rates of subsequent low-trauma fracture. Interestingly, they found that patients with prior low-trauma fractures had a greater subsequent risk of high-trauma fractures and people without prior fractures [1].

These results are similar to those reported by Mackey and colleagues in the Study of Osteoporotic Fractures in women and MrOS study in men that found that individuals who sustained a fracture carefully defined as high trauma had similar risks of subsequent fractures of any type [3]. Specifically, they had a very similar risk of low-trauma fractures than did individuals with a fracture classified as low trauma. These associations remained similar when the analysis was limited to those with more extreme, "severe trauma" such as car crashes.

Studies that have measured BMD in those with high- and low-trauma fractures have found that the associations are also very similar. In SOF and MrOS, the associations between BMD of the hip and spine and risk of fracture were very similar for both low- and high-trauma fractures, and the prevalence of osteoporosis, defined as a T-score $\leq-2.5$ in the total 
hip, was very similar for both types of fractures in both men and women [3]. In the Geelong Study, the associations between BMD of the hip, forearm, and total body were very similar between low- and high-trauma fractures, being reduced by about $0.3 \mathrm{SD}$ units as compared with people without prior fracture [4]. In a study from Sydney, the associations between BMD of the spine and hip were very similar between minimal and more than minimal trauma fractures, being reduced by about 1.0 SD units as compared with expected BMD [5].

Furthermore, in data from over 30,000 women participants in the pivotal trials of alendronate, zoledronate, and denosumab along with data from trials of lasofoxifene and clodronate, Mackey and colleagues found that the reduction in risk was identical for fractures classified as low trauma $(0.77,95 \%$ confidence interval, $0.71-0.83)$ or high trauma $(0.74,95 \%$ confidence interval, $0.57-0.96)$ by definitions used in those trials [6].

The proportion of fractures termed "high trauma" has varied considerably by study with much higher proportions of fractures - up to 50\%-classified as "high" or "moderate and high" trauma in data from hospitals and trauma centers. In contrast, the proportion of fractures termed "high trauma" has been lower-about $9 \%$ overall-in observational studies, such as SOF and MrOS, and in randomized trials where the definition of low and high trauma has been prospectively defined by protocol. Thus, a very large proportion of fractures managed in clinical settings is classified as high-trauma fractures and would be excluded from evaluation and treatment that is applied only to "low-trauma" or "fragility" fractures. This is not justified by the studies that have more rigorously defined degree of trauma.

Some might propose identifying cases of "extreme trauma" that would definitely fracture normal young bones. However, any new classification will require more detailed history and accurate estimation of extreme force applied the fracture site than was done by experts who classified trauma in the SOF and MrOS studies. It must be shown that the rare "extreme" trauma fractures are not associated with BMD or increased risk of subsequent fracture.

Continued classification of fractures as high- and lowtrauma or fragility fractures not justified will only harmfully neglect the very large number of patients who would not be evaluated or treated to prevent future fractures because their treating physicians or fracture liaison service inappropriately classifies their fracture as high trauma. This practice is particularly harmful to men whose fractures, by comparison with women, are more likely to be attributed to "high-trauma" accidents such as falls from ladders, sports, or machinery. Describing some fractures as due to trauma reinforces patients' common mistaken belief that their fractures are simply due to the trauma, not decreased bone strength, and, therefore, the patient need not consider treatment to improve bone strength.

Thus, we recommend that experts, clinicians, fracture liaison services, and guidelines abandon the classification of fractures as high- or low-trauma and fragility — or any other terms that suggest a rating of trauma. All types of fractures should be evaluated and treated without regard to trauma. Indeed, we recommend that people stop attempting to rate or record degree of trauma because such ratings are at best inaccurate and would promote the continued neglect of those patients who are misclassified as having fractures that do not warrant evaluation and treatment.

Funding information Richard Eastell received consultancy funding from IDS, Roche Diagnostics, GSK Nutrition, FNIH, Mereo, Lilly, Sandoz, Nittobo, Abbvie, Samsung, and Haoma Medica and grant funding from Nittobo, IDS, Roche, Amgen, and Alexion.

\section{Compliance with ethical standards}

Conflict of interest Steven R. Cummings received consultancy and grant funding from Amgen and Radius.

\section{References}

1. Leslie WD, Schousboe JT, Morin SN, Martineau P, Lix LM, Johansson H, McCloskey EV, Harvey NC, Kanis JA (2019) Fracture risk following high-trauma versus non-trauma fracture: a registry-based cohort study. Osteoporos Int 2020 (in press)

2. Muschitz C, Kocijan R, Baierl A, Dormann R, Feichtinger X, Haschka J, Szivak M, Muschitz GK, Schanda J, Pietschmann P, Resch H, Dimai HP (2017) Preceding and subsequent high- and low-trauma fracture patterns-a 13-year epidemiological study in females and males in Austria. Osteoporos Int 28:1609-1618

3. Mackey DC, Lui LY, Cawthon PM, Bauer DC, Nevitt MC, Cauley JA, Hillier TA, Lewis CE, Barrett-Connor E, Cummings SR, Study of Osteoporotic Fractures (SOF) and Osteoporotic Fractures in Men Study (MrOS) Research Groups (2007) High-trauma fractures and low bone mineral density in older women and men. JAMA 298: 2381-2388

4. Sanders KM, Pasco JA, Ugoni AM, Nicholson GC, Seeman E, Martin TJ, Skoric B, Panahi S, Kotowicz MA (1998) The exclusion of high trauma fractures may underestimate the prevalence of bone fragility fractures in the community: the Geelong Osteoporosis Study. J Bone Miner Res 13:1337-1342

5. Pereira L, Bliuc D, Stanford P, Eisman JA, Center JR (2017) Morethan-minimal-trauma fractures are associated with low bone density: an 8-year prospective study. Osteoporos Int 28:103-110

6. Mackey DC, Black DM, Bauer DC, McCloskey EV, Eastell R, Mesenbrink P, Thompson JR, Cummings SR (2011) Effects of antiresorptive treatment on nonvertebral fracture outcomes. J Bone Miner Res 26:2411-2418

Publisher's note Springer Nature remains neutral with regard to jurisdictional claims in published maps and institutional affiliations. 